\title{
Dolmetschen als Schlüssel zur Chancengleichheit
}

\author{
Carlos Quinto ${ }^{a}$, Robin Rieserb, Barbara Weilc
}

${ }^{a}$ Dr. med., Mitglied des FMH-Zentralvorstandes, Departementsverantwortlicher Public Health und Gesundheitsberufe; ${ }^{b}$ Wissenschaftlicher Mitarbeiter Abteilung Public Health; ' ${ }^{c}$ Leiterin Abteilung Public Health FMH

\section{Die Kommunikation zwischen medizinischem Personal und Patient ist einer der wichtigsten Faktoren, um eine erfolgreiche Behandlung gewährleisten zu können.}

Sprachliche und kulturelle Unterschiede machen einen Austausch zwischen den Beteiligten schwierig oder teilweise gar unmöglich. Die weltweite Migration führt zu einer stärkeren Durchmischung verschiedener Kulturen und Sprachen, Verständigungsprobleme, welche damit einhergehen, führen oft dazu, dass die gesundheitliche Chancengleichheit nicht mehr gewährleistet ist. Das Überbrücken dieser Barrieren geschieht im besten Fall durch professionelle Dolmetschende. Der aktuelle Stand in der Schweiz beim Dolmetschen im Gesundheitswesen wird nachfolgend beleuchtet.

\section{Vielfalt als Herausforderung}

Die Schweiz ist ein Land mit einer mehrsprachigen Tradition. Vier offizielle Landessprachen werden in unserem Land gesprochen. Es treffen Sprachkulturen aufeinander, vermischen sich oder entfernen sich voneinander. Der «Röstigraben» zwischen der Deutschschweiz und der Romandie ist das wahrscheinlich prominenteste Beispiel. In der Schweiz sprechen vier von zehn Personen einmal pro Woche zwei Sprachen, jede fünfte Person drei Sprachen und 7\% vier oder mehr Sprachen. Zu unseren Landessprachen kommen die Muttersprachen der in der Schweiz wohnhaften Ausländer dazu; fast ein Viertel der Bevölkerung stammt ursprünglich nicht aus der Schweiz. Ein Teil der zugewanderten Personen, insbesondere bildungsferne, beherrscht die Landessprachen nur teilweise oder gar nicht und ist deswegen in verschiedenen Bereichen benachteiligt. Bildung, Gesundheit und soziales Umfeld sind nur einige der betroffenen Bereiche. Unverständnis und Missverständnisse machen es den Betroffenen schwer, sich verständlich auszudrücken und für ihre Gesundheit einzustehen [1].

\section{Der Zugang für Benachteiligte ist schwieriger}

Personen, welche eine tiefe Bildung, ein niedriges Einkommen oder eine schlechte berufliche Stellung aufweisen, haben ein höheres Risiko für verschiedenste Erkrankungen sowie eine kürzere Lebenserwartung. Sie zeigen mehr gesundheitsschädliches Verhalten, treiben weniger Sport, essen ungesünder. Diese Bevölkerungsgruppe ist deshalb umso mehr auf eine gute medizinische Versorgung angewiesen. Schwierigkeiten sich auszudrücken sind in der Schweiz bei Migranten verbreitet: Knapp 10\% der Migranten der 1. Generation geben an, keine der offiziellen Landessprachen zu beherrschen. Mancherorts werden deshalb bewusst Ärzte mit ausländischen Diplomen beschäftigt, um diese Sprachbarrieren zu überwinden. In den meisten Fällen stehen sich die Parteien jedoch ohne professionelle Hilfe gegenüber. Angehörige, Bekannte oder die Kinder der Betroffenen übernehmen dann die Übersetzungsaufgabe in der Praxis. Solche Laienübersetzer sind zwar in vielen Fällen hilfreich, bringen aber auch

\section{Knapp 10\% der Migranten der 1. Generation} geben an, keine der offiziellen Landessprachen zu beherrschen.

Probleme mit sich. Unvollständige Übersetzungen, das bewusste Vorenthalten von Informationen, Auslassen von Tabus und unangenehmen Fragen können bei Laienübersetzungen dazu führen, dass unnötige Untersuchungen und Behandlungen durchgeführt werden oder angezeigte Behandlungen nicht stattfinden. Es entstehen unnötige Kosten und Frustration auf beiden Seiten. Eine Übersetzung durch professionelle Dolmetscher berücksichtigt die sprachlichen Finessen der Ursprungs- und Zielsprache, die kulturellen Kontexte 
beider Seiten und führt zu besserer Compliance, höherer Behandlungsqualität und verbesserter Patientensicherheit [1].

\section{Die Situation in der Schweiz}

Seit mehr als 15 Jahren stehen dem Gesundheitspersonal in der Schweiz Dolmetscher für Situationen schwieriger sprachlicher Verständigung zur Verfügung. Allein im Jahr 2019 wurden 163153 Dolmetschereinsätze im Gesundheitsbereich verzeichnet [2]. Eine Umfrage aus dem Jahr 2017 zeigt, dass zwei Drittel dieser Leistungen im ambulanten Setting stattfanden, mehr als die Hälfte der Haus- und Kinderärzte führen mindestens einmal im Monat Konsultationen durch, bei denen eine direkte und adäquate Kommunikation nicht

\section{Die FMH fordert die Behebung der Unklar- heiten in der Organisation und Finanzierung von Dolmetschleistungen.}

möglich ist. Die Problematik zeigt sich auch darin, dass 44\% der Haus- und Kinderärzte oder -ärztinnen nicht wissen, wie solche Einsätze organisiert werden können. Weiter besteht bei 53\% der Haus- und Kinderärztinnen oder -ärzten Unklarheit darüber, wer die Finanzierung dieser Angebote übernimmt. Die grösste Hürde ist die Organisation solcher Einsätze, welche von 58\% der Haus- und Kinderärzte als mühsam wahrgenommen werden.

Dazu kommt, dass die Finanzierung in den Kantonen unterschiedlich geregelt wird. Nur jeder zehnte befragte Arzt kannte die allenfalls vorhandenen Finanzierungsmöglichkeiten solcher Dolmetscheinsätze. Waren diese bekannt, stieg die Wahrscheinlichkeit, die Dolmetschangebote zu nutzen, um das Achtfache an. Leider ist in der Regel keine Finanzierung sichergestellt. Die meisten Einsätze von Dolmetschenden finden aktuell im ambulanten Setting statt. Eine Finanzierung ist im TARMED nicht enthalten, könnte aber im TARDOC zwischen der Tarifpartnern verhandelt und eingeführt werden. Ein weiteres grosses Hindernis sind die aktuell im TARMED bestehenden Zeitlimitationen. Der Bundesrat ist der Ansicht, dass die Finanzierung zwischen den Tarifparteien geregelt werden müsse und sieht keinen Handlungsbedarf, müsste aber deshalb endlich konsequenterweise TARDOC genehmigen [3].

\section{Was geschieht jetzt?}

Wenig erstaunlich ist deshalb in Anbetracht der oben genannten Situation, dass erst kürzlich zwei Motionen $[4,5]$ dem Bundesrat vorgelegt wurden, welche das Thema Dolmetschen in der ambulanten Patientenversorgung zur Sprache bringen. Die FMH fordert die Behebung der Unklarheiten in der Organisation und Finanzierung von Dolmetschleistungen. Eine landesweit einheitliche Regelung und Organisation ist notwendig, um in allen Regionen den Zugang zu Dolmetschleistungen und damit eine qualitativ gute, kostengünstigere und faire medizinische Versorgung zu ermöglichen. In der Bundesverfassung (Art. 41, Abs. 1b) wird festgehalten, dass sich Bund und Kantone einsetzen, dass jede Person die für ihre Gesundheit notwendige Pflege erhält [6]. Das Bundesamt für Gesundheit (BAG) empfiehlt für emotionale und komplexe Gespräche mit allophonen Patienten Dolmetschende beizuziehen [7]. Aus alle diesen Gründen ist es an der Zeit, dass Bund und Kantone handeln, um die Versorgung fremdsprachiger Patienten in der Schweiz zu verbessern und um der Ärzteschaft und medizinischem Personal die nötige Unterstützung bieten zu können.

Literatur

1 Jaeger F. Dolmetschende in Schweizer Haus- und Kinderarztpraxen. Binningen; 2017. https://kollegium.ch/sites/default/ files/20170824_Dolmetschende_d.pdf

2 INTERPRET. Einsatzstatistik zum interkulturellen Dolmetschen und Vermitteln 2019. 2020. www.inter-pret.ch/admin/data/files/ marginal_asset/file/489/2019_einsatzstatistik_dt. pdf?lm=1605533382.

3 Jaeger $\mathrm{F}$ et al. Barriers to and Solutions for Addressing Insufficient Professional Interpreter Use in Primary Healthcare. BMC Health Services Research. 2019;19(1):753. https://doi.org/10.1186/s12913-019 4628-6.

$4 \quad 19.4279 \rightarrow$ Notwendige Dolmetscherdienste in der Arztpraxis und im Ambulatorium $\rightarrow$ Geschäft $\rightarrow$ Das Schweizer Parlament (parlament.ch).

$5 \quad$ 19.4357 $\rightarrow$ Ärztinnen und Ärzte und Patientinnen und Patienten. Verstehen und verstanden werden. Finanzierung von interkulturellem Dolmetschen im ambulanten Bereich $\rightarrow$ Geschäft $\rightarrow$ Das Schweizer Parlament (parlament.ch).

6 SR 101 Bundesverfassung der Schweizerischen Eidgenossenschaft vom 18. April 1999 (admin.ch).

7 www.bag.admin.ch/bag/de/home/strategie-und-politik/nationale-gesundheitsstrategien/gesundheitliche-chancengleichheit/ interkulturelles-dolmetschen.html. 